\title{
VISUAL RESPONSE PROPERTIES IN THE TECTORECIPIENT ZONE OF THE CAT'S LATERAL POSTERIOR-PULVINAR COMPLEX: A COMPARISON WITH THE SUPERIOR COLLICULUS ${ }^{1}$
}

\author{
LEO M. CHALUPA, ${ }^{2}$ ROBERT W. WILLIAMS ${ }^{3}$ AND MICHAEL J. HUGHES \\ Department of Psychology and The Physiology Graduate Group, University of California, Davis, California 95616
}

Received April 4, 1983; Revised June 16, 1983; Accepted June 28, 1983

\begin{abstract}
The medial portion of the cat's lateral posterior-pulvinar complex $(\mathrm{LPm})$ receives a prominent ascending projection from the superficial layers of the superior colliculus. This region of the thalamus has been suggested to serve as a relay by which visual information from the midbrain could be conveyed to extrastriate cortex. In order to determine how the functional organization within the LPm compares with that of the superior colliculus, visual response properties of LPm and superior collicular neurons were examined under identical experimental conditions. The majority of neurons in the LPm, as in the superior colliculus, respond vigorously to moving stimuli, and a substantial proportion of these cells also exhibit a preference for movements in a particular direction. Furthermore, most cells in the LPm, in common with those of the tectum, respond only in a phasic manner to flashed stimuli, have homogeneous receptive field organization, and show response suppression to stimuli larger than the activating region of the receptive field. As in the colliculus, the ipsilateral visual field is represented in the LPm. In spite of these similarities, there are also some striking differences between the visual responses of LPm and collicular neurons. First, the average size of receptive fields of neurons within the LPm is at least twice that of units in the superficial gray layers of the tectum. Second, more cells in the colliculus are directionally selective than those in the LPm, and the distribution of preferred directions is different in the two regions. Third, an appreciable proportion (27\%) of the cells in the LPm are orientation selective, whereas this response property is only rarely encountered in the cat's tectum. Fourth, many LPm neurons can only be activated by binocular stimulation, whereas most collicular units respond equally well to stimulation of either eye. Collectively, these findings indicate that there is a substantial transformation in the lateral posterior-pulvinar complex of the ascending visual influx provided by the superior colliculus.
\end{abstract}

On the basis of afferent connection patterns, the lateral posterior (LP)-pulvinar complex of the cat is subdivided into three broad zones. The first, and most lateral region, the pulvinar proper, receives a direct projection from the retina (Berman and Jones, 1977; Kawamura et al., 1980; Leventhal et al., 1980), cortical area 19 (Kawamura et al., 1974; Updyke, 1977), and the pretectum (Graybiel, 1972a, b, c; Berson and Graybiel, 1978). The

\footnotetext{
${ }^{1}$ This work was supported by Grant EY-03491 from the National Eye Institute. We thank Dr. Chris Johnson for determining the luminance of stimuli employed in these experiments, Dr. Larry Butcher for his advice regarding the acetylcholinesterase histochemistry protocol, and Dr. Lamberto Maffei for his helpful comments on the manuscript.

${ }^{2}$ To whom correspondence should be addressed.

${ }^{3}$ Present address: Section of Neuroanatomy, Yale University School of Medicine, 333 Cedar Street, New Haven CT 06510.
}

second zone, medial to the pulvinar, is the lateral portion of the LP which receives projections from cortical areas 17, 18, and 19 (Kawamura et al., 1974; Updyke, 1981). The third zone, medial to the principal corticorecipient region, is innervated by ascending projections originating from the superficial layers of the superior colliculus. Each of these regions of the LP-pulvinar complex projects to largely nonoverlapping portions of the visual cortex (Graybiel, 1972a, b; Hughes, 1980; Graybiel and Berson, 1981; Symonds et al., 1981).

Although a number of studies have dealt with the visual responses of LP-pulvinar neurons (e.g., Godfraind et al., 1972; Veraart et al., 1972; Chalupa and Fish, 1978; Mason, 1978, 1981; Fish and Chalupa, 1979), we still do not know how the functional organization within the three visual zones relates to their principal sources of 
visual input. In this context we have been particularly interested in the tectorecipient zone of the LP-pulvinar complex. This region, which has been designated the LPm (Graybiel, 1972c), consists of a broad wedge of cells situated just lateral to the pretectum and extending as far forward as the anterior third of the lateral geniculate body. The connectivity pattern suggests that the LPm could serve to relay visual information from the superior colliculus to the cortex; however, functional validation for this viewpoint is surprisingly limited.

The visual receptive field properties of the cat's superior colliculus have been well documented (for recent review see Chalupa, 1984). Furthermore, Mcllwain (1978) has shown that the tectal neurons which can be activated antidromically by stimulation of the posterior thalamus do not differ appreciably in terms of their visual receptive field characteristics from the overall population. Therefore, it seemed pertinent to inquire how the visual response properties of LPm neurons compare to those of the superior colliculus. In the present study we have addressed this issue by studying the visual responses of LPm and collicular neurons under identical conditions.

\section{Materials and Methods}

Animal preparation. Experiments were carried out on 17 adult cats. Each animal was premedicated with atropine $(0.08 \mathrm{mg})$ and anesthetized for surgery with ketamine hydrobromide $(15 \mathrm{mg} / \mathrm{kg})$. The saphenous vein was cannulated, a tracheotomy was performed, and the animal was placed in a stereotaxic instrument. A craniotomy was made over the posterior thalamus and, in some cases, over the midbrain. Two stainless steel screws, which served as EEG electrodes, were embeddded bilaterally in the frontal sinus, and a head mount apparatus was cemented with dental acrylic to the anterior portion of the skull. The dura was reflected and, for the duration of the experiment, the cortex was covered with a mixture of mineral oil and petroleum jelly (Vaseline). Heart rate was monitored during surgery and throughout the recording session. The ear and eye bars were removed and the animal's head was shifted forward to the edge of the stereotaxic frame which provided an unobstructed view of the tangent screen placed $57 \mathrm{~cm}$ in front of the animal's eyes.

The pupils were dilated with a drop of $1 \%$ atropine sulfate, and the nictitating membranes were retracted with phenylephrine hydrochloride. Clear plastic lenses were applied to prevent corneal drying. Animals were paralyzed with gallamine triethiodide (infusion rate of $10 \mathrm{mg} / \mathrm{kg} / \mathrm{hr}$ ) and ventilated artificially with a mixture of $70 \%$ nitrous oxide and $30 \%$ oxygen. The end-tidal $\mathrm{CO}_{2}$ level was monitored continuously with a Beckman LB-2 medical gas analyzer and maintained between 3.5 and $4.5 \%$. Normal body temperature was stabilized using a circulating heated water pad. An intravenous injection of $20 \mathrm{mg} / \mathrm{kg}$ of chloralose was administered about $1 \mathrm{hr}$ before recordings were begun, and this was repeated approximately every $8 \mathrm{hr}$ during the 2 -day recording session. In this preparation the cortical electroencepha- logram consisted of slow-wave activity interrupted intermittently by brief spindle bursts.

Visual stimulation and recordings. The optic disks were projected and plotted on the tangent screen using the method of Fernald and Chase (1971). The luminance of the tangent screen was $0.16 \mathrm{~cd} / \mathrm{m}^{2}$ and the stimuli employed were 1.9 log units above background. Occasionally, dark stimuli were employed. In these instances the background was changed to $6.7 \mathrm{~cd} / \mathrm{m}^{2}$ and the luminance of the stimulus was $1.6 \mathrm{log}$ units below background.

Extracellular single unit recordings were carried out in the conventional manner using varnished tungsten microelectrodes with an impedance of 10 megohms measured at $1 \mathrm{kHz}$. Only units with biphasic potentials and a minimum waveform duration of $1 \mathrm{msec}$, assumed to be of somal origin (Hubel, 1960), were studied.

For each visually responsive cell, we first plotted the activating region of the receptive field. This was done by presenting a moving or flashing stimulus well outside the receptive field while gradually approaching its center. Usually the smallest spot size which elicited reliable responses was employed for this purpose. Some cells responded poorly to flashing or moving spots but could be activated vigorously by slits of light with a particular orientation. The borders between responsive and unresponsive regions were approximated by straight lines drawn directly onto the tangent screen. Receptive field position in the visual field was referenced to the projection of area centralis as calculated from the data of Bishop et al. (1962).

An automated visual stimulator, controlled by the experimenter or by computer, was used to test the following response properties. (a) Directional sclectivity was assessed by moving a spot of light across the receptive field vertically, horizontally, and along two oblique axes. (b) Orientation specificity was tested by flashing a bar of light, $1.5^{\circ}$ in width and between 5 and $40^{\circ}$ in length, at different angles. At each orientation the position of the bar was systematically varied within the field. In all cases, responses to a moving slit of light were compared to those of a spot with a diameter equal to the length of the bar. The size of the spot was always kept smaller than the receptive field. (c) Cutoff velocity was defined as the highest speed capable of modulating the neuronal discharge. This value was determined by gradually increasing and then decreasing the velocity of an optimal stimulus. (d) The internal organization of the receptive field was studied by flashing stimuli throughout the activating region. The response polarity $(\mathrm{ON}, \mathrm{OFF}$, or $\mathrm{ON}-\mathrm{OFF}$ ) was initially determined with the smallest spot centered on the field which evoked reliable activity. To test for the presence of surround suppression, the spot size was increased in several steps until its dimension exceeded that of the activating region. $(e)$ Ocular dominance was appraised by stimulating each eye separately with a stimulus optimal for evoking binocular responses.

Responses were usually judged by listening to the audio monitor. However, for many cells, poststimulus time histograms were made on-line to verify the qualitative impressions and to document particular examples of receptive field properties. 
Small electrolytic lesions ( $5 \mu \mathrm{A}$ for $5 \mathrm{sec}$ ) were made along each electrode track. At the end of the experiment animals were deeply anesthetized and were perfused transcardially with cold solutions of neutral $0.9 \%$ saline and $10 \%$ formalin. The brain was blocked immediately and sectioned at $50 \mu \mathrm{m}$.

Acetylcholinesterase histochemistry and verification of recording sites. The $\mathrm{LPm}$ region cannot be adequately differentiated from other portions of the LP-pulvinar complex in Nissl-stained sections. However, Graybiel and Berson (1980) have shown that the tectorecipient zone of the LP-pulvinar complex can be identified by its rich content of acetylcholinesterase. In the present study we have taken advantage of this finding to determine whether our recordings were within the LPm. For this purpose, alternate sections were reacted to demonstate acetylcholinesterase content using the direct coloring method of Karnovsky and Roots (1964) as modified by Butcher et al. (1974) or stained with cresyl violet. Electrode penetrations were reconstructed by drawing the Nissl-stained sections which showed the marking lesions using a microscope fitted with a drawing attachment.
The LPm was delineated from adjacent histochemically processed sections. The locus of every cell for which data are reported was verified histologically.

\section{Results}

A detailed description of the pattern of acetylcholinesterase label in the cat's thalamus has been provided by Graybiel and Berson (1980). They have shown that the medial portion of the LP-pulvinar complex, which stains heavily for cholinesterase, corresponds closely to the tectorecipient zone. Figure 1 is composed of a set of frontal sections through the posterior thalamus reacted to demonstrate the distribution of acetylcholinesterase activity. The LPm stands out as an obliquely oriented wedge of dense label which extends from the dorsomedial to the ventrolateral portion of the posterior thalamus. We are confident that all neurons were recorded in the portion of the LP-pulvinar complex distinguished by high cholinesterase activity. Their distribution is shown in Figure 2.

Under the experimental conditions employed in this study, $93 \%$ of the LPm cells (256 of 276 ) were found to
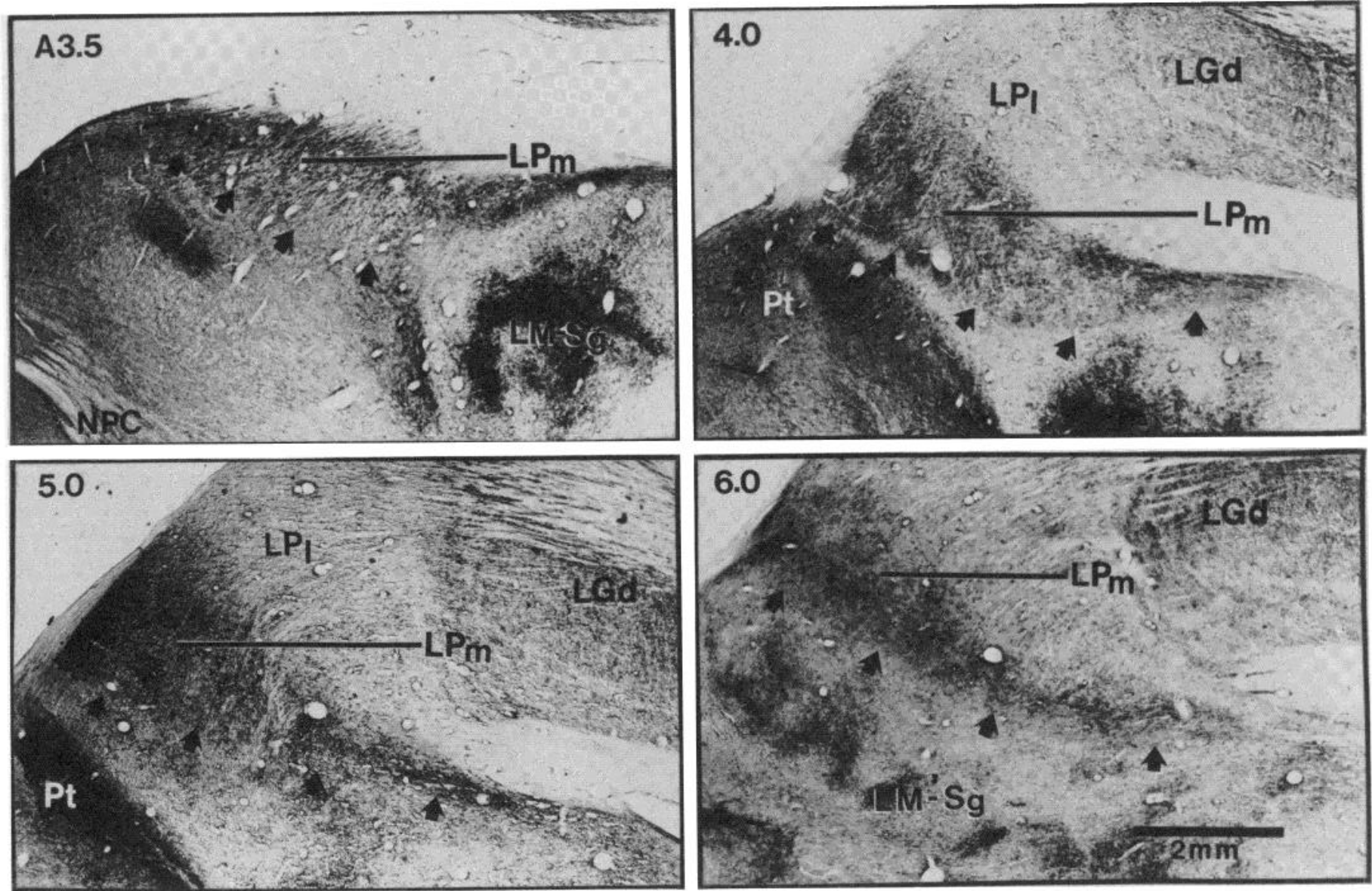

Figure 1. Photomicrographs of four coronal sections through the posterior thalamus stained for acetylcholinesterase. The arrows denote the lower border of the LPm. The Horsley-Clarke frontal coordinate of each section is indicated by the number in the upper left corner of the photograph. $L P_{m}$, nucleus lateralis posterior, pars medialis; $L P_{l}$, nucleus lateralis posterior, pars lateralis; $L M$, nucleus lateralis medialis; $S g$, suprageniculate nucleus; $L G d$, dorsal nucleus of the lateral geniculate body; $P t$, pretectal region. 


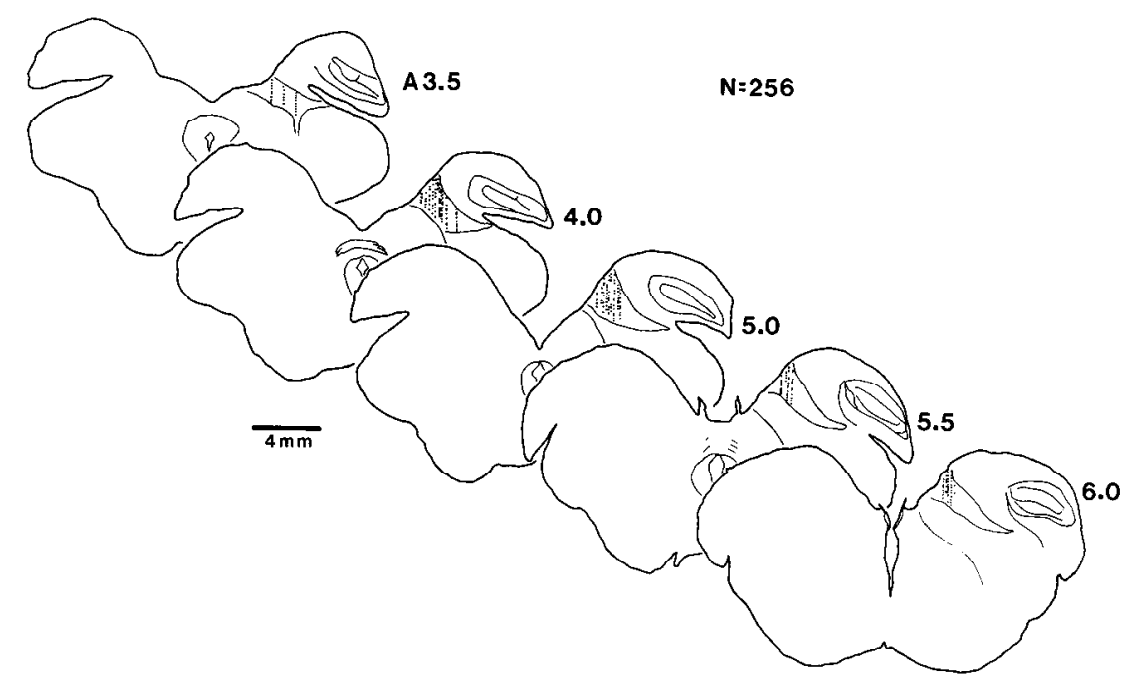

Figure 2. The loci in the LPm of the 256 visually responsive cells whose response properties were examined in this study. The number to the right of each section refers to the corresponding Horsley-Clarke frontal coordinate.

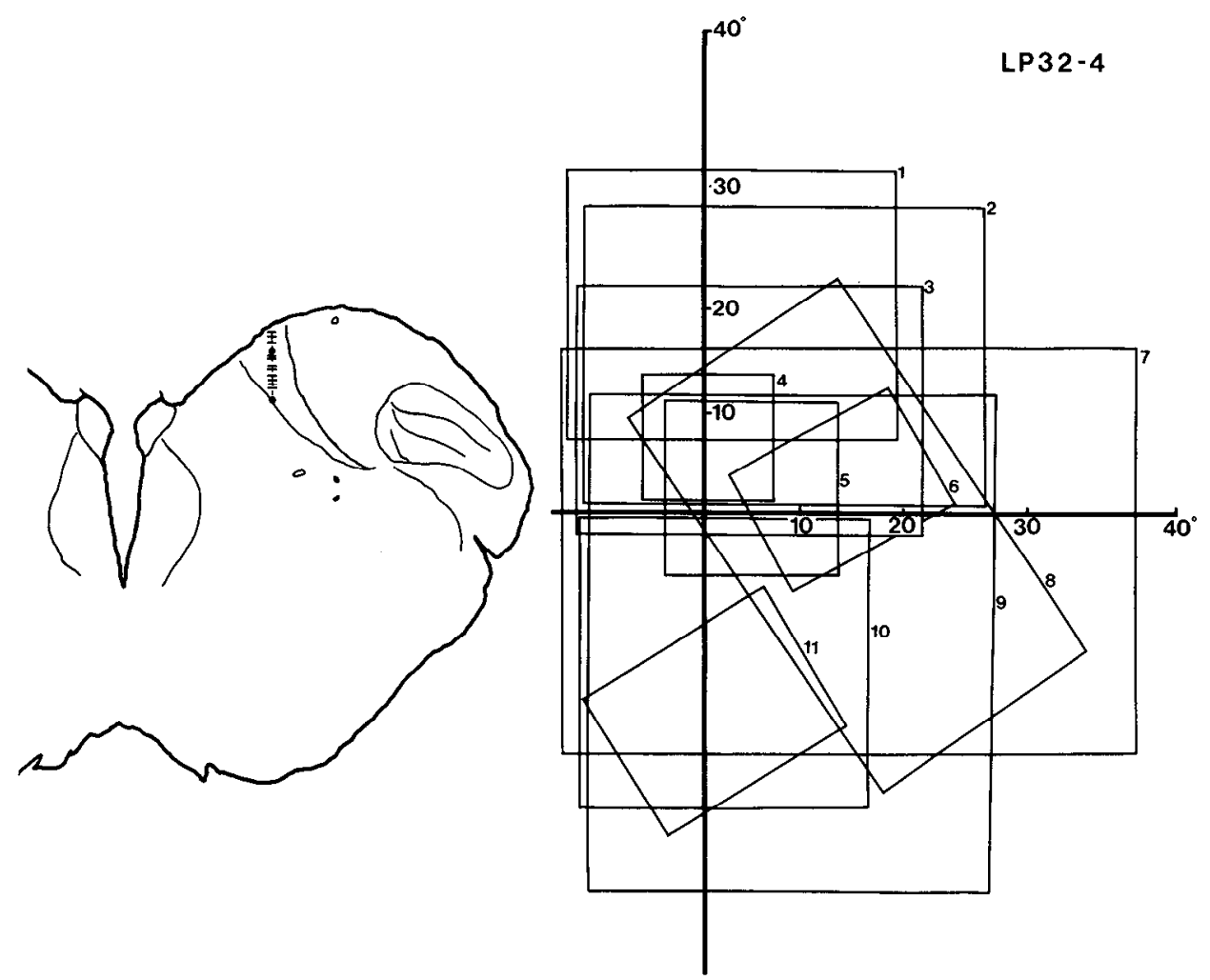

Figure 3. Visual receptive fields of 11 cells isolated along one penetration through the LPm. The 1st receptive field was plotted for a neuron isolated in the most dorsal portion of the track and the 11th was plotted for a neuron at the bottom of the penetration. The vertical and horizontal meridians are indicated by heavy lines. The hatch marks in the LPm denote the loci of these cells, and the two solid circles along the penetration depict the marking lesions.

be responsive to visual stimuli. An overall topographic organization was apparent, confirming previous reports (Mason, 1978, 1981; Raczkowski and Rosenquist, 1981). Within the caudal portion of the LPm all receptive fields were above the horizontal meridian. Similarly, the most superficial neurons in the central portion of the tectorecipient zone also had receptive fields above the horizontal meridian, but the elevation of fields decreased as the electrode progressed ventrally. Only lower fields were represented in the anterior segment of the LPm. How- 
ever, it was not uncommon to find some disorder in the overall retinotopic representation outlined above. In a number of cases the receptive field centers of two cclls separated by less than $100 \mu \mathrm{m}$ differed by more than $20^{\circ}$.

Receptive field dimensions. Figure 3 shows the receptive fields of 11 cells isolated along one penetration through the LPm. This track passed through the middle portion of the LPm and clearly illustrates the progressive downward shift of receptive fields. Many fields cross the vertical meridian, in some instances by as much as $30^{\circ}$. Although all of the receptive fields were quite large, it may he seen in Figure 3 that there is a tendency for the smaller fields to be situated near the area centralis representation. This relation was examined in more detail by plotting the field size as a function of the distance from area centralis (Fig. 4). Although considerable variability exists at any given eccentricity, there is a clear trend for receptive field area to increase with eccentricity. The correlation between receptive field size and eccentricity was 0.50 , which is statistically significant $(t=8.6, p<0.01)$. It is remarkable that, even within $10^{\circ}$ of the area centralis representation, fields typically encompass an area of $391 \mathrm{deg}^{2}$, and this figure almost doubles $\left(695 \mathrm{deg}^{2}\right)$ at eccentricities between 11 and $20^{\circ}$. Beyond $20^{\circ}$ most fields were enormous, with average areas above $800 \mathrm{deg}^{2}$.

Degree of binocularity. The ocular dominance scale of Hubel and Wiesel (1962) was used to assess the degree

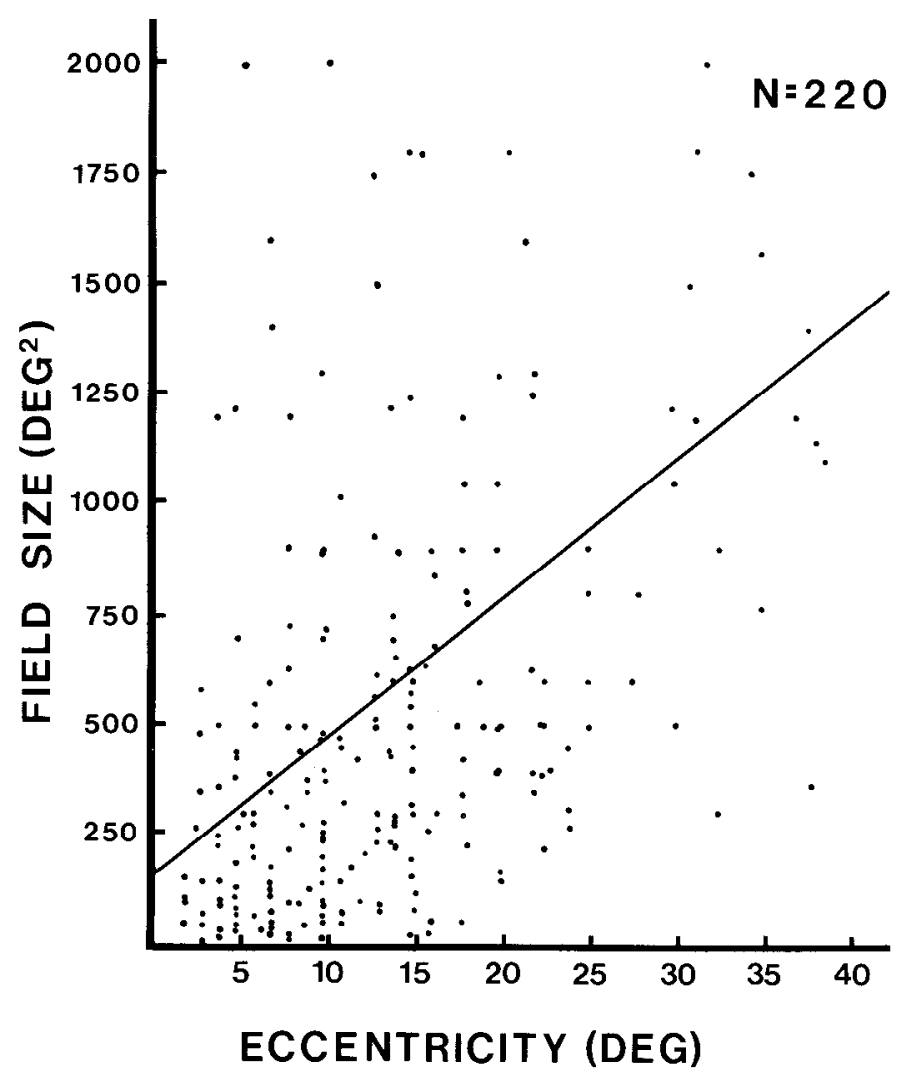

Figure 4. Receptive field area as a function of eccentricity (distance of receptive field center from the area centralis representation) for 220 cells in the LPm. The regression line was fit by the least squares method. It has a slope of 32.6 and a $y$ intercept of $140 \mathrm{deg}^{2}$.

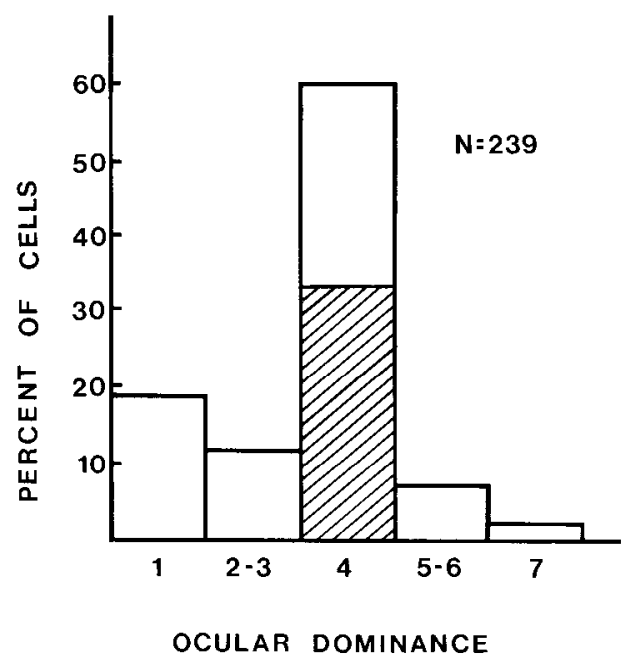

Figure 5. The ocular dominance of 239 cells in the LPm. Cells in group 1 responded only to stimulation of the contralat eral eye and those in group 7 could only be activated by the ipsilateral eye. Group 4 cells responded equally well to stimulation of either eye, and included units which could only be activated by binocular stimulation. This subgroup is indicated by the lined portion of the bar.
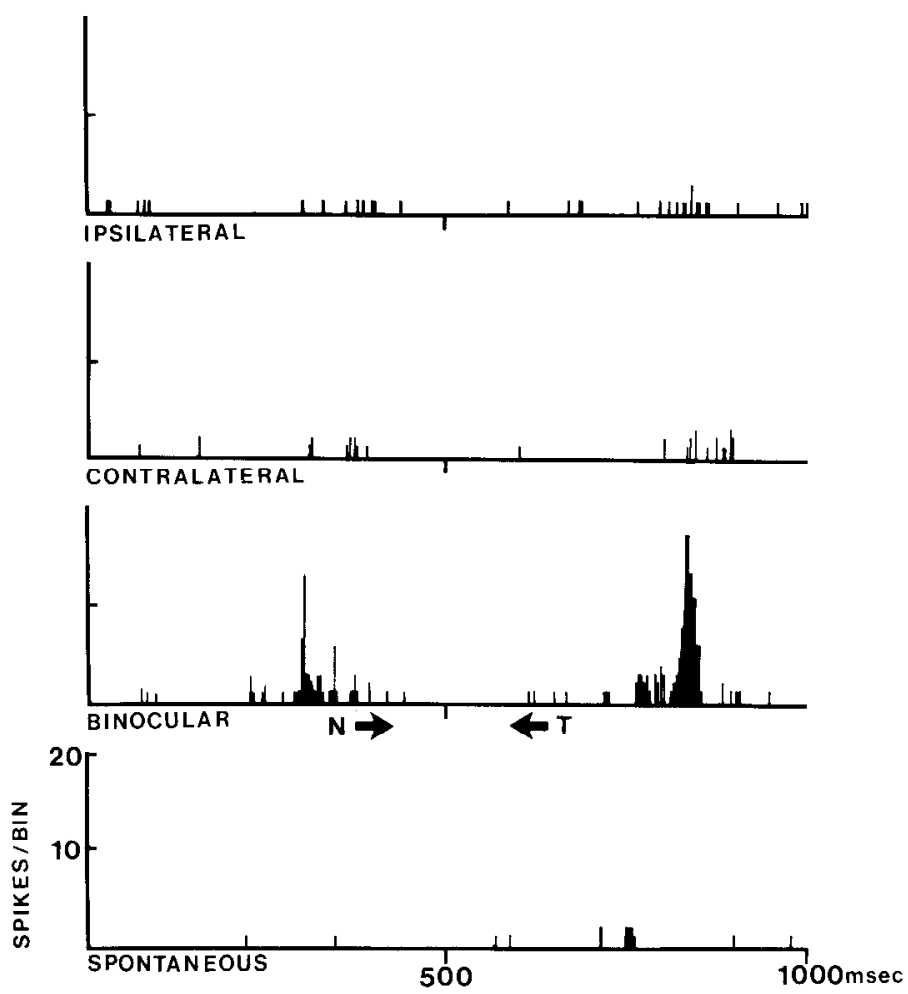

Figure 6. An example of a cell which responded only to binocular stimulation. The stimulus was a bar of light which moved nasally and temporally across the receptive field at a speed of $240^{\circ}$ sec. Each poststimulus time histogram (PSTH) is based on 20 stimulus presentations, and the bin width is 4 msec.

of binocularity of 239 units (Fig. 5). Monocular cells comprise $21 \%$ of the sample, and the great majority of these (19\% of the total) responded only to stimulation of the contralateral eye. Almost $60 \%$ of the binocular 
cells were activated equally by stimulation of either eye. However, virtually all binocular cells responded much more vigorously to binocular than to monocular stimulation. In many instances $(34 \%, n=81)$, binocular activation was essential to evoke a consistent response. An example of this intriguing phenomenon is shown in Figure 6. Cells which respond only to binocular stimulation have been noted previously in the inferior pulvinar of the monkey (Bender, 1982) and in the cat's lateral suprasylvian visual area (Spear and Baumann, 1975) to which the LPm is reciprocally connected (Symonds et al., 1981; Updyke, 1981). However, the proportion of such neurons appears to be much greater in the LPm than that which has been reported in any other visual area.

Responses to movement. Most cells in the LPm $(n=$ 221) could be activated by moving stimuli which were smaller than the activating region of the receptive field. These movement-sensitive units responded over a wide velocity range, and more than $80 \%$ of the cells could be activated by stimulus speeds in excess of $400^{\circ} / \mathrm{sec}$. Half of the cells $(49 \%, n=125)$ showed a clear response asymmetry to different directions of stimulus movement. We considered a cell to be directionally selective if there

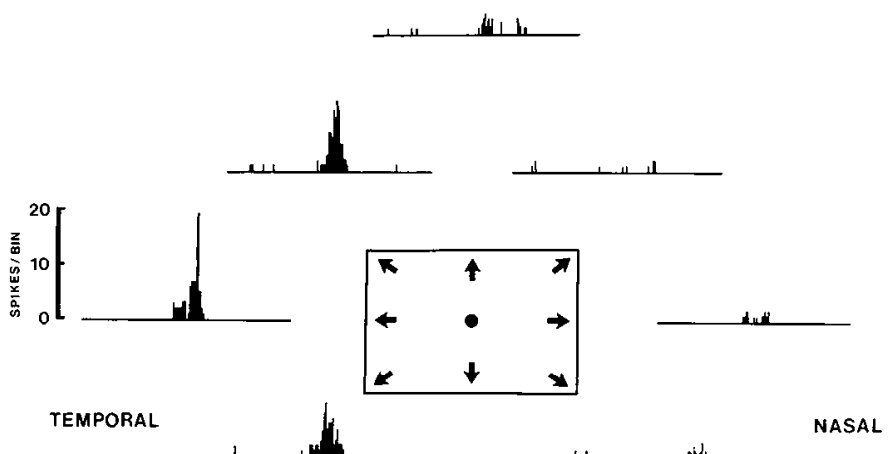

A
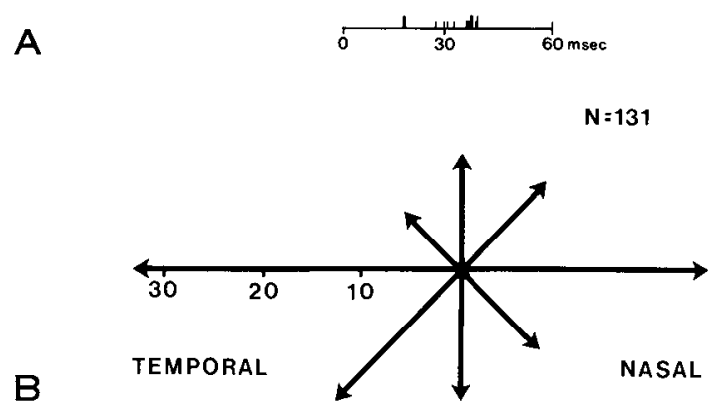

Figure 7. Directionally selective cells. A, Responses of a typical directionally selective cell in the LPm. The different directions of stimulus movement are indicated by the arrows which point to the corresponding PSTHs. The diameter of the spot was $10^{\circ}$ and the speed of movement was $200^{\circ} / \mathrm{sec}$. The dimensions of this receptive field are $20 \times 30^{\circ}$. The PSTHs are based on 20 stimulus presentations, and the bin width is 4.8 msec. $B$, The distribution of the preferred directions of the 131 directionally selective cells in the LPm. Temporal is toward the periphery of the contralateral visual field, away from area centralis. The length of the arrows is scaled to the number of cells which preferred a given direction of stimulus movement. was at least a 2 to 1 difference in the number of action potentials evoked by stimulus movement in two opposing directions. Generally, only stimuli which were above background were employed to assess this response property, but in cases where dark spots were also used, contrast reversal did not change the preferred direction. All of these directionally selective cells were broadly tuned for this stimulus parameter. A typical example of the response pattern of one such cell is illustrated in Figure $7 A$, and in this case the unit responded to any stimulus with a temporal component. We considered the preferred direction to be that which was in the mid-range of the stimulus directions capable of activating the cell. Figure $7 B$ shows the distribution of preferred directions, and a clear bias for stimulus movement in the horizontal plane is apparent. Furthermore, a slight majority of units were more sensitive to stimuli moved into the temporal periphery of the contralateral visual field than toward the center of gaze. The preferred directions of these cclls were not related in any obvious manner to the location of receptive fields within the visual field.

Orientation specificity. We were surprised to find that a substantial proportion (27\%) of the LPm cells were clearly selective for stimulus orientation. These cells tended to have the smallest receptive fields with an average field area of $337 \mathrm{deg}^{2}$. In contrast, the mean field size of the nonoriented units was $743 \mathrm{deg}^{2}$. Figure $8 A$ shows the responses of one oriented cell, and Figure $8 B$ depicts the overall distribution of the preferred orientations. A clear bias in favor of vertically oriented stimuli is apparent. However, it should be noted that more than half of the oriented units were directionally selective (33 of 61), and in all but one instance the preferred stimulus orientation was orthogonal to the optimal direction of movement. Thus, in this subpopulation of neurons, direction and orientation specificity are largely interdependent, and therefore, given the pronounced bias for movement in the horizontal plane, the results depicted in Figure $8 B$ are not unexpected. Parenthetically, all orientation-selective cells which also displayed directional specificity responded more vigorously to bars than to spots of light.

Receptive field organization and surround suppression. Although movement was generally the most effective stimulus for activating LPm neurons, most also responded reliably to small flashing spots of light. In all instances only phasic responses were obtained. The majority of cells yielded OFF responses $(55 \%, n=106)$, a few units $(10 \%, n=19)$ gave only $\mathrm{ON}$ discharges, whereas the remainder $(35 \%, n=67)$ responded both to stimulus onset and offset. In all but five cells, the discharge polarity evoked by a small flashing spot was constant irrespective of the stimulus position within the receptive field. In these five atypical units, two or more well defined subregions yielded responses of opposite polarity to those obtained from the central portion of the receptive field.

When the size of the stimulus exceeded the dimensions of the activating region of the receptive field, $36 \%$ of the cells $(n=60)$ showed no evidence for an inhibitory surround mechanism, and of these, a few $(n=16)$ showed response summation (Fig. 9A). However, in the majority of units $(64 \%, n=106)$, a suppressive surround could be 

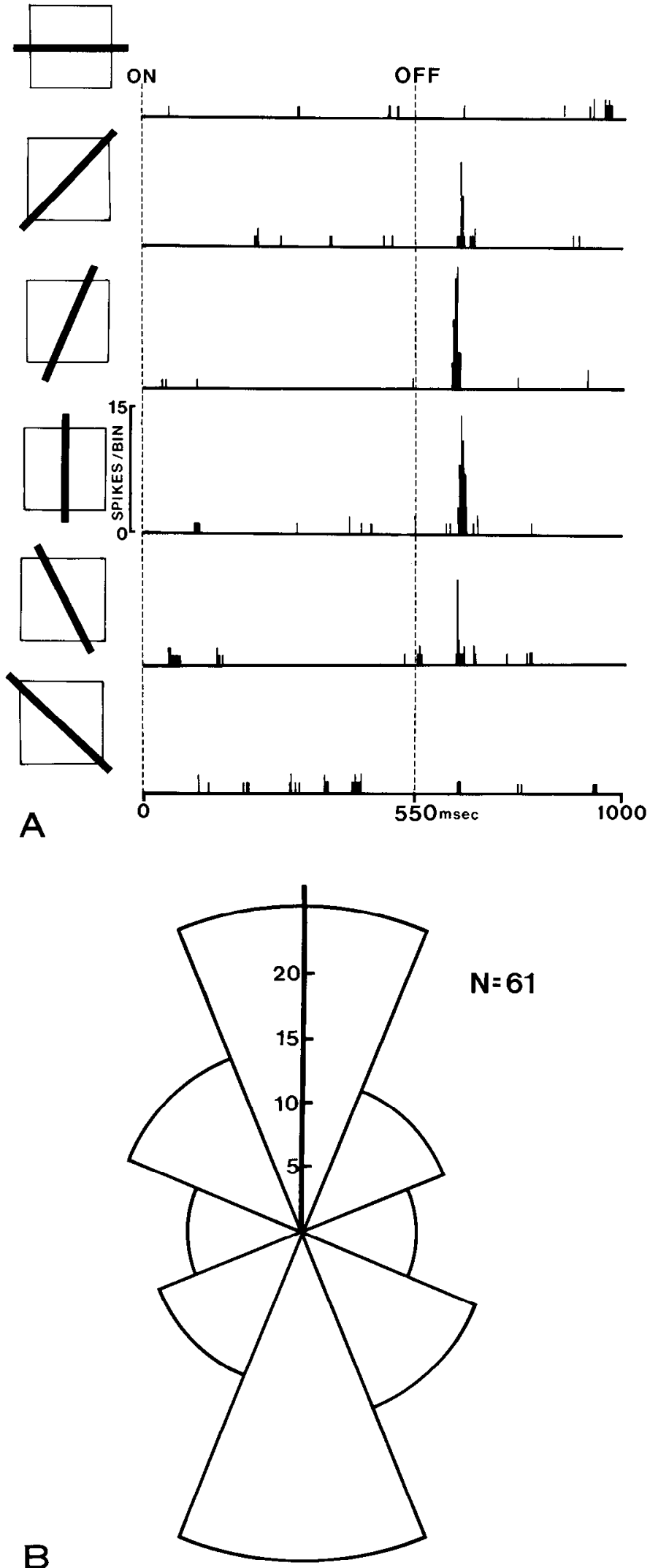

Figure 8. Orientation selectivity. A, An example of an oricntation-selective cell in the LPm. The orientation of the flashing bar is shown to the left of the corresponding PSTHs which are based on 20 stimulus presentations and a bin width of $4 \mathrm{msec}$. $B$, The distribution of the preferred orientations of the 61 oriented cells isolated in the LPm. clearly demonstrated (Fig. 9, $B$ and $C$ ), even though responses of opposite polarity were not obtained from outside the field center. In extreme cases simultaneous activation of the field center and surround completely abolished neuronal responsivity (Fig. 9R). Some ONOFF units displayed selective suppression since the large stimulus attenuated only the $\mathrm{ON}$ or the OFF responses, whereas the other discharge pattern was largely unaffected (Fig. 9C). Cells with similar integrative properties have been documented in the mammalian tectum (Cynader and Berman, 1972; Updyke, 1974; Berman and Cynader, 1975; Rhoades and Chalupa, 1977; Chalupa and Rhoades, 1978).

Recordings from the superior colliculus. In spite of the fact that the visual receptive field properties of the cat's superior colliculus have been examined under a variety of experimental conditions, there is a remarkable degree of general consensus regarding the functional organization of this structure (for recent reviews see Chalupa, 1984; Stein, 1984). Nevertheless, we thought it important to examine the visual responses of superior collicular neurons with procedures identical to those used to study the tectorecipient zone. For this reason, in three of the animals used for LPm recordings, the visual responses of 55 neurons in the superior colliculus were also examined. All of these units were in the superficial laminae of the superior colliculus and had receptive fields within $40^{\circ}$ of the area centralis representation. The distribution of superior collicular receptive fields within the visual field did not differ appreciably from those of LPm neurons. The tectal cells responded briskly to moving stimuli smaller than the activating region of the receptive field, and $78 \%$ (40 of 51 units tested) showed a marked preference for the direction of stimulus movement. There was a pronounced bias (35 of 40 cells) for stimulus movement with a temporal component; that is, into the periphery of the contralateral visual field. Only two units in the colliculus were orientation selective, one of which was very broadly tuned for this stimulus parameter. Thirty-seven collicular cells responded reliably to small flashing spots, and $73 \%$ of these units demonstrated response suppression when the dimensions of the stimulus exceeded those of the receptive field-activating region. The average receptive field area of superior collicular neurons was less than one-half that of LPm cells (312 $\mathrm{deg}^{2}$ in the colliculus and $634 \mathrm{deg}^{2}$ in the LPm).

\section{Discussion}

Largely on the basis of anatomical evidence, the tectorecipient zone of the LP-pulvinar complex has been considered to serve as a relay by which visual information from the superior colliculus is conveyed to extrastriate cortex (e.g., Graybiel, 1972a, b, c; Jones, 1974; Diamond, 1976; Chalupa, 1977). In this context, it is important to compare the functional organization of the LPm with that of the superior colliculus. In making this comparison we will rely upon our own observations, which are in close agreement with those of others who have studied the visual physiology of the cat's superior colliculus in greater detail (e.g., Mcllwain and Buser, 1968; Sterling and Wickelgren; 1969; Rosenquist and Palmer, 1971; Berman and Cynader, 1972; Stein and Arigbede, 1972; 

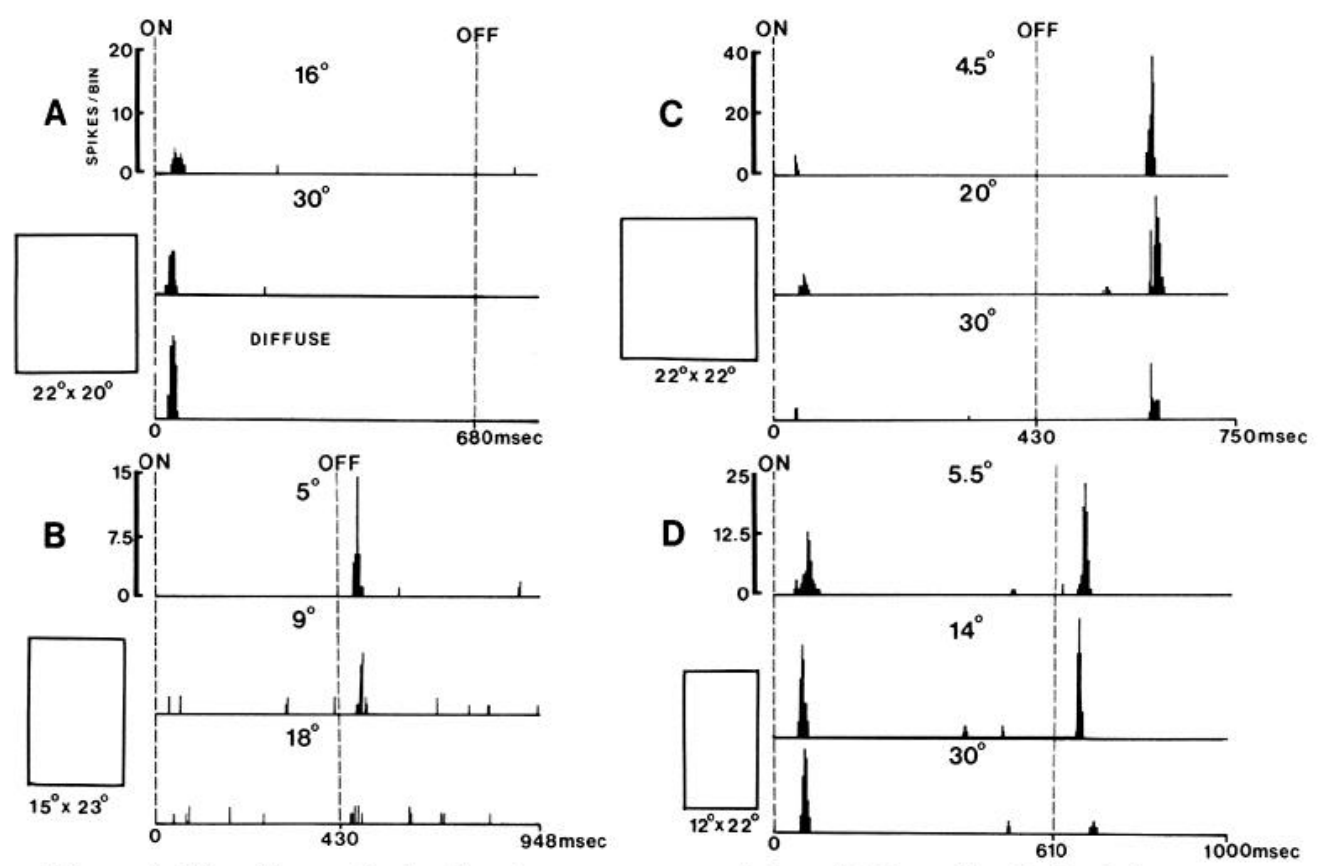

Figure 9. The effects of stimulus size upon responsivity of LPm cells. Each of these neurons had a homogeneously organized activating region. The stimuli were centered on the field. In $A$, response summation is evident even when the diffuse stimulus, which encompassed most of the tangent screen, was employed. In $B$, complete response suppression may be seen when the size of the stimulus exceeded the dimensions of the activating region. In $C$, a partial response suppression is illustrated. In $D$, the $\mathrm{ON}$ discharge is unaffected by the largest stimulus, but the OFF response is completely suppressed. The diameter of the flashing spot is denoted above each PSTH and the dimensions of the receptive field-activating region are shown to the right of each set of PSTHs. The PSTHs are based on 20 stimulus presentations and the bin widths are 4 mesc.

Dreher and Hoffmann, 1973; McIlwain, 1978; Stein, 1978; reviewed in Chalupa, 1984).

Certain receptive field properties are prominent in the $\mathrm{LPm}$ and in the superior colliculus. Visual cells in both structures are highly sensitive to moving stimuli, and a substantial proportion of these units are selective for the direction of stimulus movement. Furthermore, all of the neurons in the LPm and in the colliculus which can be activated by flashing stimuli respond only in a phasic manner. The internal organization of the receptive field of these neurons is homogeneous; that is, the same type of discharge pattern is elicited at all sites within the activating region. Stimuli larger than the activating region produce a marked response attenuation in many LPm and tectal units. In addition, the ipsilateral visual field is represented in the LPm as in the colliculus. These similarities seem to support the idea that the LPm region conveys visual information from the tectum to extrastriate cortex in a relatively unmodified manner. However, as described in the following section, this is not the case, since there are striking differences in the functional organization of these two regions.

A comparison of response properties. One of the most obvious contrasts between neurons of the LPm and the colliculus is their receptive field size. The mean size of fields within the LPm is at least twice that of units within the superficial strata of the superior colliculus. This expansion could arise through the convergence of tectal efferents upon LPm neurons. In support of this notion, it has been reported (Caldwell and Mize, 1981) that focal injection of peroxidase enzyme into the tectorecipient zone labeled cells which were distributed quite broadly in the colliculus. Nonetheless, the pattern of label reflected a coarse grained visuotopy described in earlier anatomical studies (Kawamura, 1974; Kawamura and Kobayashi, 1975) and by this and previous electrophysiological investigations (Mason, 1978, 1981; Raczkowski and Rosenquist, 1981).

Second, proportionally more cells in the tectum are directionally selective than in the LPm, and the distributions of preferred directions are markedly different. The vast majority of collicular units preferred movement in the horizontal plane toward the periphery. In contrast, in the LPm, only a small bias for such movement was apparent. This comparative symmetry could be due to a "balancing" input from the contralateral colliculus (Niimi et al., 1970). However, the number of collicular cells which project across the midline is minute (B. P. Abramson and L. M. Chalupa, manuscript in preparation), so that it is unlikely that this connection could explain the substantial proportion of LPm cells which prefer movement into the ipsilateral visual field.

Third, about $26 \%$ of the units in the LPm are orientation selective, whereas this response property is rarely encountered in the cat's superior colliculus. It is conceivable that this characteristic could be synthesized intrinsically by the tectorecipient neurons, much as do the neurons of area 17 from an ascending geniculate input. It should also be noted that the tectorecipient zone of the LP-pulvinar receives descending projections from the 
lateral suprasylvian area (Updyke, 1981), as well as from a recently described visual region in the anterior ectosylvian sulcus (Olson and Graybiel, 1981). This cortical influx could contribute to the organization of certain visual response properties within the IAPm, although oriented cells are not commonly encountered in the lateral suprasylvian visual area (Spear and Baumann, 1975).

Fourth, whereas most neurons of the superior colliculus respond equally well to stimulation of either eye, more than $30 \%$ of the LPm cells require binocular stimulation. Monocular stimulation of either eye is highly ineffective in activating these cells. Neurons such as we have described in the LPm may provide a neural correlate for a purely binocular visual mechanism described in recent psychophysical experiments (Wolfe and Held, 1981). Thus, there are clearly two types of binocular cells: an inclusive variety which responds to stimulation of either eye, and an exclusive variety which is activated only by binocular input. Both types are present in the tectorecipient region of the LP-pulvinar complex.

Tectal neurons which project to the LPm are located in the deep subdivisions of the superficial gray and the upper portion of the stratum opticum (Kawamura et al., 1980; Caldwell and Mize, 1981). These strata receive predominantly a Y-type retinal input (Itoh et al., 1981) which may account for the very high cutoff velocities of I.Pm neurons. On the other hand, it should be noted that the morphology of tecto- $\mathrm{LPm}$ cells is quite diverse and includes: stellate, vertical fusiform, granular, and horizontal neurons (Caldwell and Mize, 1981). This anatomical diversity may be related to McIlwain's (1978) observation that units in the superficial laminae of the superior colliculus with ascending projections to the LPpulvinar complex form a heterogeneous group with respect to their response properties. A thorough description of receptive field types as a function of laminar position within the superficial layers of the cat's colliculus would provide further insight into the nature of the information transfer between tectum and LPm. Such an analysis has been carried out in the rabbit (Graham et al., 1981) and tree shrew (Albano et al., 1978) but, surprisingly, not in the cat.

Nevertheless, the results of the present study allow certain conclusions to be drawn regarding the functional significance of this tectothalamic system. The similarities which we have noted between the visual response properties of LPm and collicular neurons suggest that the LPm, like the colliculus, may be part of an ascending system concerned with localization and fixation of visual targets (Peck et al., 1980). On the other hand, the differences we have noted, including larger receptive fields, selectivity to stimulus orientation, a balanced distribution of the preferred axis of stimulus movement, and the discovery of neurons with purely binocular receptive fields within the LPm, point to a more complex role for this region in visual function. Although the nature of this role is unknown, the present results suggest a substantial transformation within the LPm of information provided by the ascending projection from the midbrain. This may involve the integration of tectal and cortical visual inputs. In this context it would be of interest to determine how the response properties of LPm neurons are modified by the selective inactivation of the cortical and tectal afferents.

\section{References}

Albano, J. E., A. L. Humphrey, and T. T. Norton (1978) Laminar organization of receptive-field properties in tree shrew superior colliculus. J. Neurophysiol. 41: 1140-1164.

Bender, D. B. (1982) Receptive field properties of neurons in the macaque inferior pulvinar. J. Neurophysiol. 48: 1-17.

Berman, N., and M. Cynader (1972) Comparison of receptivefield organization of the superior colliculus in Siamese and normal cats. J. Physiol. (Lond.) 224: 363-389.

Berman, N., and M. Cynader (1975) Receptive fields in cat superior colliculus after visual cortex lesions. J. Physiol. (Lond.) 245: 261-270.

Berman, N., and E. G. Jones (1977) A retinopulvinar projection in the cat. Brain Res. 131: 237-218.

Berson, D. M., and A. M. Graybiel (1978) Parallel thalamic zones in the LP-pulvinar complex of the cat identified by their afferent and efferent connections. Brain Res. 147: 139148.

Bishop, P. O., W. Kozak, and G. J. Vakkur (1962) Some quantitative aspects of the cat's eye: Axis and plane of reference, visual field coordinates and optics. J. Physiol. (Lond.) 163: 466-502.

Butcher, L. L., S. M. Eastgate, and G. K. Hodge (1974) Evidence that punctate intracerebral administration of 6-hydroxydopamine fails to produce selective neuronal degeneration: Comparison with copper sulfate and factors governing the deportment of fluids injected into brain. Naunyn-Schmiedebergs Arc. Pharmacol. 285: 31-70.

Caldwell, R. B., and R. R. Mize (1981) Superior colliculus neurons which project to the cat lateral posterior nucleus have varying morphologies. J. Comp. Neurol. 203: 53-66.

Chalupa, L. M. (1977) A review of cat and monkey studies implicating the pulvinar in visual function. Behav. Biol. 20: 149-167.

Chalupa, L. M. (1984) Visual physiology of the mammalian superior colliculus. In Comparative Neurology of the Optic Tectum, H. Vanegas, ed., Plenum Press, New York, in press.

Chalupa, L. M., and S. E. Fish (1978) Response characteristics of visual and extra-visual neurons in the pulvinar and lateral posterior nuclei of the cat. Exp. Neurol. 36: 96-120.

Chalupa, L. M., and R. W. Rhoades (1978) Modification of visual response properties in the superior colliculus of the golden hamster following stroboscopic rearing. J. Physiol. (Lond.) 274: 571-592.

Cynader, M., and N. Berman (1972) Receptive-field organization of monkey superior colliculus. J. Neurophysiol. 35: 187201.

Diamond, I. T. (1976) Organization of visual cortex: Comparative anatomical and behavioral studies. Fed. Proc. 35: 6067.

Dreher, B., and K. -P. Hoffmann (1973) Properties of excitatory and inhibitory regions in the receptive fields of single units in the cat's superior colliculus. Exp. Brain Res. 16: 333-353.

Fernald, R., and R. Chase (1971) An improved method for plotting retinal landmarks and focusing the eyes. Vision Res. 11: 95-96.

Fish, S. E., and L. M. Chalupa (1979) Functional properties of pulvinar-lateral posterior neurons which receive input from the superior colliculus. Exp. Brain Res. 36: 245-257.

Godfraind, J. M., M. Meulders, and C. Veraart (1972) Visual properties of neurons in pulvinar, nucleus lateralis posterior and nucleus suprageniculatus thalami in the cat. I. Qualitative investigation. Brain Res. 44: 503-526.

Graham, J., H. E. Pearson, N. Berman, and E. H. Murphy 
(1981) Laminar organization of superior colliculus in the rabbit: A study of receptive-field properties of single units. J. Neurophysiol. 45: 915-932.

Graybiel, A. M. (1972a) Some ascending connections of the pulvinar and nucleus lateralis posterior of the thalamus in the cat. Brain Res. 44: 99-125.

Graybiel, A. M. (1972b) Some extrageniculate visual pathways in the cat. Invest. Ophthalmol. 11: 322332.

Graybiel, A. M. (1972c) Some fiber pathways related to the posterior thalamic region in the cat. Brain Behav. Evol. 6: 363-393.

Graybiel, A. M., and D. M. Berson (1980) Histochemical identification and afferent connections of subdivisions in the lateralis-posterior pulvinar complex and related nuclei in the cat. Neuroscience 5: 1175-1238.

Graybiel, A. M., and D. M. Berson (1981) On the relation between transthalamic and transcortical pathways in the visual cortext. In The Organization of Cerebral Cortex, F. O., Schmitt, F. G. Worden, and F. Dennis, eds., pp. 285-319, MIT Press, Cambridge, MA.

Hubel, D. H. (1960) Single unit activity in lateral geniculate body and optic tract of unrestrained cats. J. Physiol. (Lond.) 150: 91-104.

Hubel, D. H., and T. N. Wiesel (1962) Receptive fields, binocular interaction and functional architecture in the cat's visual cortex. J. Physiol. (Lond.). 160: 106-154.

Hughes, H. C. (1980) Efferent organization of the cat pulvinar complex, with a note on bilateral claustrocortical and reticulocortical connections. J. Comp. Neurol. 193: 937-963.

Itoh, K., M. Conley, and I. T. Diamond (1981) Different distribution of large and small retinal ganglion cells in the cat after HRP injections of single layers of the lateral geniculate body and the superior colliculus. Brain Res. 207: 147-152.

Jones, E. G. (1974) The anatomy of extrageniculate visual mechanisms. In The Neurosciences: Third Study Program, F. O. Schmitt and F. G. Worden, eds., pp. 215-227, MIT Press, Cambridge, MA.

Karnovsky, M. J., and L. Roots (1964) A "direct-coloring" thiocholine method for cholinesterases. J. Histochem. Cytochem. 12: 219-221.

Kawamura, S. (1974) Topical organization of the extrageniculate visual system in the cat. Exp. Neurol. 45: 451-461.

Kawamura, S., and E. Kobayashi (1975) Identification of laminar origin of some tecto-thalamic fibers in the cat. Brain Res. 91: 281-285.

Kawamura, S., J. M. Sprague, and K. Niimi (1974) Corticofugal projections from the visual cortices to the thalamus, pretectum and superior colliculus in cat. J. Comp. Neurol. 158: $339-362$.

Kawamura, S., N. Ukushima, S. Hattori, and M. Kudo (1980) Laminar segregation of cells of origin of ascending projections from the superficial layers of the superior colliculus in the cat. Brain Res. 184: 486-490.

Leventhal, A. G., J. Keens, and I. Tork (1980) The afferent ganglion cells and cortical projections of the retinal recipient zone (RRZ) of the cat's "pulvinar complex." J. Comp. Neurol. 194: 535-554.

Mason, R. (1978) Functional organization in the cat's pulvinar complex. Exp. Brain Res. 31: 51-66.

Mason, R. (1981) Differential responsiveness of cells in the visual zones of the cat's LP-pulvinar complex to visual stimuli. Exp. Brain Res. 43: 25-33.

McIlwain, J. T. (1978) Properties of cells projecting rostrally from the superficial layers of the cat's superior colliculus. Brain Res. 143: 445-457.

Mcllwain, J. T., and P. Buser (1968) Receptive fields of single cells in the cat's superior colliculus. Exp. Brain Res. 5: 314325.

Niimi, K., M. Miki, and S. Kawamura (1970) Ascending projections of the superior colliculus in the cat. Okajimas Folia Anat. Jpn. 47: 269-287.

Olson, C. R., and A. M. Graybiel (1981) A visual area in the anterior ectosylvian sulcus of the cat. Soc. Neurosci. Abstr. 7: 831.

Peck, C. K., M. Schlag-Rey, and J. Schlag (1980) Visuo-oculomotor properties of cells in the superior colliculus of the alert cat. J. Comp. Neurol. 194: 97-116.

Raczkowski, D., and A. C. Rosenquist (1981) Retinotopic organization in the cat lateral posterior-pulvinar complex. Brain Res. 221: 185-191.

Rhoades, R. W., and L. M. Chalupa (1977) Differential effects of stimulus size on "on" and "off" responses of superior collicular neurons. Fxp. Neurol. 57: 57-66.

Rosenquist, A. C., and L. A. Palmer (1971) Visual receptivefield properties of cells in the superior colliculus after cortical lesions in the cat. Exp. Neurol. 33: 629-652.

Spear, P. D., and T. D. Baumann (1975) Receptive-field characteristics of single neurons in lateral suprasylvian visual area of the cat. J. Neurophysiol. 38: 1403-1420.

Stein, B. E. (1978) Nonequivalent visual, auditory, and somatic corticotectal influences in cat. J. Neurophysiol. 41: 55-64.

Stein, B. E. (1984) Development of the superior colliculus. Annu. Rev. Neurosci., in press.

Stein, B. E., and M. O. Arigbede (1972) Unimodal and multimodal response properties of neurons in the cat's superior colliculus. Exp. Neurol. 36: 179-196.

Sterling, P., and B. Wickelgren (1969) Visual receptive fields in the superior colliculus of the cat. J. Neurophysiol. 32: 115.

Symonds, L. L., A. C. Rosenquirt, S. B. Edwards, and L. A. Palmer (1981) Projections of the pulvinar-lateral posterior complex to visual cortical areas in the cat. Neuroscience 6 : 1995-2020.

Updyke, B. V. (1974) Characteristics of unit responses in superior colliculus in the Cebus monkey. J. Neurophysiol. 37: 896-909.

Updyke, B. V. (1977) Topographic organization of the projections from cortical areas 17,18 , and 19 onto the thalamus, pretectum, and superior colliculus in the cat. J. Comp. Neurol. 173: 81-121.

Updyke, B. V. (1981) Projections from visual areas of the middle suprasylvian sulcus onto the lateral posterior complex and adjacent thalamic nuclei in cat. J. Comp. Neurol. 201: 477-506.

Veraart, C., M. Meulders, and J. M. Godfraind (1972) Visual properties of neurons in pulvinar, nucleus lateralis posterior and nucleus suprageniculatus thalami in the cat. II. Quantitative investigation. Brain Res. 44: 527-546.

Wolfe, J. M., and R. Held (1981) A purely binocular mechanism in human vision. Vision Res. 21: 1755-1759. 